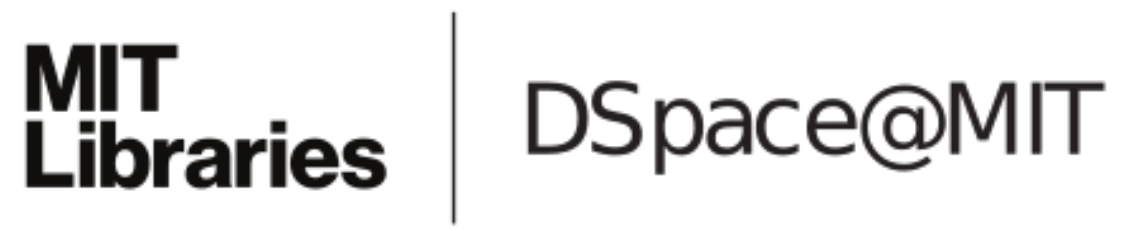

\author{
MIT Open Access Articles
}

\section{Inference for Parameters Defined by Moment Inequalities: A Recommended Moment Selection Procedure}

The MIT Faculty has made this article openly available. Please share how this access benefits you. Your story matters.

Citation: Donald W. K. Andrews, Panle Jia Barwick. “Inference for Parameters Defined by Moment Inequalities: A Recommended Moment Selection Procedure." Econometrica 80.6 (2012): 2805-2826.

As Published: http://dx.doi.org/10.3982/ECTA8166

Publisher: Econometric Society

Persistent URL: http://hdl.handle.net/1721.1/73649

Version: Author's final manuscript: final author's manuscript post peer review, without publisher's formatting or copy editing

Terms of use: Creative Commons Attribution-Noncommercial-Share Alike 3.0 


\title{
Inference for Parameters Defined by Moment Inequalities: A Recommended Moment Selection Procedure
}

\author{
Donald W. K. Andrews* \\ Cowles Foundation for Research in Economics \\ Yale University \\ Panle Jia \\ Department of Economics \\ MIT \\ June 2008 \\ Revised: May 2011
}

*Andrews gratefully acknowledges the research support of the National Science Foundation via grant SES-0751517. The authors thank Steve Berry for numerous discussions and comments. The authors also thank the co-editor, three referees, and Michael Wolf for very helpful comments. 


\begin{abstract}
This paper is concerned with tests and confidence intervals for parameters that are not necessarily identified and are defined by moment inequalities. In the literature, different test statistics, critical value methods, and implementation methods (i.e., the asymptotic distribution versus the bootstrap) have been proposed. In this paper, we compare these methods. We provide a recommended test statistic, moment selection critical value method, and implementation method. We provide data-dependent procedures for choosing the key moment selection tuning parameter $\kappa$ and a size-correction factor $\eta$.
\end{abstract}

Keywords: Asymptotic size, asymptotic power, bootstrap, confidence set, generalized moment selection, moment inequalities, partial identification, refined moment selection, test, unidentified parameter.

JEL Classification Numbers: C12, C15. 


\section{Introduction}

This paper considers inference in moment inequality models with parameters that need not be identified. We focus on confidence sets for the true parameter, as opposed to the identified set. We construct confidence sets (CS's) by inverting Anderson-Rubintype test statistics. We consider a class of such statistics and a class of generalized moment selection (GMS) critical values. This approach follows Imbens and Manski (2004), Chernozhukov, Hong, and Tamer (2007) (CHT), Andrews and Guggenberger (2009) (AG), Andrews and Soares (2010) (AS), and other papers.

GMS and subsampling tests and CS's are the only methods in the literature that apply to arbitrary moment functions and have been shown to have correct asymptotic size in a uniform sense, see AG, AS, and Romano and Shaikh (2008). AS and Bugni (2010) show that GMS tests dominate subsampling tests in terms of asymptotic size and power properties. In addition, in our experience based on simulation results, subsampling tests often are substantially under-sized in finite samples in moment inequality testing problems. Hence, we focus on GMS critical values.

GMS tests and CS's depend on a test statistic function $S$, a critical value function $\varphi$, and a tuning parameter $\kappa$. In this paper we determine a combination that performs well in terms of size and power and can be recommended for general use. To do so, we consider asymptotics in which $\kappa$ equals a finite constant plus $o_{p}(1)$, rather than asymptotics in which $\kappa \rightarrow \infty$ as $n \rightarrow \infty$, as has been considered elsewhere in the literature. ${ }^{1}$

We find that an adjusted Gaussian quasi-likelihood ratio (AQLR) test statistic combined with a "t-test moment selection" critical value performs very well in terms of asymptotic average power compared to other choices considered in the literature. ${ }^{2}$ We develop data-dependent methods of selecting $\kappa$ and a size-correction factor $\eta$ and show that they yield very good asymptotic and finite-sample size and power. We provide a table that makes them easy to implement in practice for up to ten moment inequalities.

We show that with i.i.d. observations bootstrap critical values out-perform those based on the asymptotic-distribution in terms of finite-sample size. We also show that the bootstrap version of the AQLR test performs similarly in terms of null rejection probabilities and power to an analogous test based on the empirical likelihood ratio (ELR) statistic. The AQLR-based test is noticeably faster to compute than the ELR-

\footnotetext{
${ }^{1}$ The theoretical arguments mentioned in the preceding paragraph rely on $\kappa \rightarrow \infty$ asymptotics.

${ }^{2}$ The "adjustment" in the AQLR test statistic is designed to handle singular asymptotic correlation matrices of the sample moment functions.
} 
based test and avoids computational convergence problems that can arise with the ELR statistic when the correlation matrix of the moment conditions is singular.

The asymptotic results of the paper apply to i.i.d. and time series data and to moment functions that are based on preliminary estimators of point-identified parameters.

In short, the contribution of this paper relative to the literature is to compare moment inequality tests, determine a recommended test, and provide data-dependent tuning parameters.

The remainder of the paper is organized as follows. Section 2 introduces the model and describes the recommended confidence set and test. Section 3 defines the different test statistics and critical values that are compared in the paper. Section 4 provides the numerical comparisons of the tests based on asymptotic average power. Section 5 describes how the recommended data-dependent tuning parameter $\widehat{\kappa}$ and size-correction factor $\widehat{\eta}$ are determined and provides numerical results assessing their performance. Section 6 gives finite-sample results.

Andrews and Jia (2008) (AJ2) provides Supplemental Material that includes: (i) the asymptotic results that are utilized in this paper, (ii) details concerning the numerical results given here, and (iii) additional numerical results.

Let $R_{+}=\{x \in R: x \geq 0\}, R_{++}=\{x \in R: x>0\}, R_{+, \infty}=R_{+} \cup\{+\infty\}$, $K^{p}=K \times \ldots \times K$ (with $p$ copies) for any set $K$, and $0_{p}=(0, \ldots, 0)^{\prime} \in R^{p}$.

\section{Model and Recommended Confidence Set}

The moment inequality model is specified as follows. The true value $\theta_{0}\left(\in \Theta \subset R^{d}\right)$ is assumed to satisfy the moment conditions:

$$
E_{F_{0}} m_{j}\left(W_{i}, \theta_{0}\right) \geq 0 \text { for } j=1, \ldots, p
$$

where $\left\{m_{j}(\cdot, \theta): j=1, \ldots, p\right\}$ are known real-valued moment functions and $\left\{W_{i}: i \geq 1\right\}$ are i.i.d. or stationary random vectors with joint distribution $F_{0}$. The observed sample is $\left\{W_{i}: i \leq n\right\}$. The true value $\theta_{0}$ is not necessarily identified. The results also apply when the moment functions in (2.1) depend on a parameter $\tau$, i.e., when they are of the form $\left\{m_{j}\left(W_{i}, \theta, \tau\right): j \leq p\right\}$, and a preliminary consistent and asymptotically normal estimator $\widehat{\tau}_{n}\left(\theta_{0}\right)$ of $\tau$ exists, see AJ2. In addition, the asymptotic results in AJ2 allow for moment equalities as well as moment inequalities. 
We are interested in tests and confidence sets (CS's) for the true value $\theta_{0}$. We consider a confidence set obtained by inverting a test. The test is based on a test statistic $T_{n}\left(\theta_{0}\right)$ for testing $H_{0}: \theta=\theta_{0}$. The nominal level $1-\alpha$ CS for $\theta$ is

$$
C S_{n}=\left\{\theta \in \Theta: T_{n}(\theta) \leq c_{n}(\theta)\right\}
$$

where $c_{n}(\theta)$ is a data-dependent critical value. ${ }^{3}$

We now describe the recommended test statistic and critical value. The justifications for these recommendations are described below and are given in detail in AJ2. The recommended test statistic is an adjusted quasi-likelihood ratio (AQLR) statistic, $T_{A Q L R, n}(\theta)$, that is a function of the sample moment conditions, $n^{1 / 2} \bar{m}_{n}(\theta)$, and an estimator of their asymptotic variance, $\widehat{\Sigma}_{n}(\theta)$ :

$$
\begin{aligned}
T_{A Q L R, n}(\theta) & =S_{2 A}\left(n^{1 / 2} \bar{m}_{n}(\theta), \widehat{\Sigma}_{n}(\theta)\right) \\
& =\inf _{t \in R_{+, \infty}^{p}}\left(n^{1 / 2} \bar{m}_{n}(\theta)-t\right)^{\prime} \widetilde{\Sigma}_{n}^{-1}(\theta)\left(n^{1 / 2} \bar{m}_{n}(\theta)-t\right), \text { where } \\
\bar{m}_{n}(\theta) & =\left(\bar{m}_{n, 1}(\theta), \ldots, \bar{m}_{n, p}(\theta)\right)^{\prime}, \bar{m}_{n, j}(\theta)=n^{-1} \sum_{i=1}^{n} m_{j}\left(W_{i}, \theta\right) \text { for } j \leq p, \\
\widetilde{\Sigma}_{n}(\theta) & =\widehat{\Sigma}_{n}(\theta)+\max \left\{\varepsilon-\operatorname{det}\left(\widehat{\Omega}_{n}(\theta)\right), 0\right\} \widehat{D}_{n}(\theta), \varepsilon=.012, \\
\widehat{D}_{n}(\theta) & =\operatorname{Diag}\left(\widehat{\Sigma}_{n}(\theta)\right), \widehat{\Omega}_{n}(\theta)=\widehat{D}_{n}^{-1 / 2}(\theta) \widehat{\Sigma}_{n}(\theta) \widehat{D}_{n}^{-1 / 2}(\theta),
\end{aligned}
$$

and $\operatorname{Diag}(\Sigma)$ denotes the diagonal matrix based on the matrix $\Sigma .{ }^{4}$ Note that the weight matrix $\widetilde{\Sigma}_{n}(\theta)$ depends only on $\widehat{\Sigma}_{n}(\theta)$ and hence $T_{A Q L R, n}(\theta)$ can be written as a function of $\left(\bar{m}_{n}(\theta), \widehat{\Sigma}_{n}(\theta)\right)$. The function $S_{2 A}(\cdot)$ is an adjusted version of the QLR function $S_{2}(\cdot)$ that appears in Section 3. The adjustment is designed to handle singular variance matrices. Specifically, the matrix $\widetilde{\Sigma}_{n}(\theta)$ equals the asymptotic variance matrix estimator $\widehat{\Sigma}_{n}(\theta)$ with an adjustment that ensures that $\widetilde{\Sigma}_{n}(\theta)$ is always nonsingular and is invariant to scale changes in the moment functions. The matrix $\widehat{\Omega}_{n}(\theta)$ is the correlation matrix that corresponds to $\widehat{\Sigma}_{n}(\theta)$.

\footnotetext{
${ }^{3}$ When $\theta$ is in the interior of the identified set, it may be the case that $T_{n}(\theta)=0$ and $c_{n}(\theta)=0$. In consequence, it is important that the inequality in the definition of $C S_{n}$ is $\leq$, not $<$.

${ }^{4}$ The constant $\varepsilon=.012$ was determined numerically based on an average asymptotic power criterion. See Section 6.2 of AJ2 for details.
} 
When the observations are i.i.d. and no parameter $\tau$ appears, we take

$$
\begin{aligned}
\widehat{\Sigma}_{n}(\theta) & =n^{-1} \sum_{i=1}^{n}\left(m\left(W_{i}, \theta\right)-\bar{m}_{n}(\theta)\right)\left(m\left(W_{i}, \theta\right)-\bar{m}_{n}(\theta)\right)^{\prime}, \text { where } \\
m\left(W_{i}, \theta\right) & =\left(m_{1}\left(W_{i}, \theta\right), \ldots, m_{p}\left(W_{i}, \theta\right)\right)^{\prime} .
\end{aligned}
$$

With temporally dependent observations or when a preliminary estimator of a parameter $\tau$ appears, a different definition of $\widehat{\Sigma}_{n}(\theta)$ often is required, see AJ2. For example, with dependent observations, a heteroskedasticity and autocorrelation consistent (HAC) estimator may be required.

The test statistic $T_{A Q L R, n}(\theta)$ is computed using a quadratic programming algorithm. Such algorithms are built into GAUSS and Matlab. They are very fast even when $p$ is large. For example, to compute the AQLR test statistic 100,000 times takes 2.6, 2.9, and 4.7 seconds when $p=2,4$, and 10, respectively, using GAUSS on a PC with a 3.4 GHz processor. ${ }^{5}$

A moment selection critical value that utilizes a data-dependent tuning parameter $\widehat{\kappa}$ and size-correction factor $\widehat{\eta}$ is referred to as a refined moment selection (RMS) critical value. Our recommended RMS critical value is

$$
c_{n}(\theta)=c_{n}(\theta, \widehat{\kappa})+\widehat{\eta}
$$

where $c_{n}(\theta, \widehat{\kappa})$ is the $1-\alpha$ quantile of a bootstrap (or "asymptotic normal") distribution of a moment selection version of $T_{A Q L R, n}(\theta)$ and $\widehat{\eta}$ is a data-dependent size-correction factor. For i.i.d. data, we recommend using a nonparametric bootstrap version of $c_{n}(\theta, \widehat{\kappa})$. For dependent data, either a block bootstrap or an asymptotic normal version can be applied.

We now describe the bootstrap version of $c_{n}(\theta, \widehat{\kappa})$. Let $\left\{W_{i, r}^{*}: i \leq n\right\}$ for $r=1, \ldots, R$ denote $R$ bootstrap samples of size $n$ (i.i.d. across samples), such as nonparametric i.i.d. bootstrap samples in an i.i.d. scenario or block bootstrap samples in a time series scenario, where $R$ is large. Define the bootstrap variance matrix estimator $\widehat{\Sigma}_{n, r}^{*}(\theta)$ as $\widehat{\Sigma}_{n}(\theta)$ is defined (e.g., as in (2.4) in the i.i.d. case) with $\left\{W_{i, r}^{*}: i \leq n\right\}$ in place of $\left\{W_{i}: i \leq n\right\}$ throughout. ${ }^{6}$ The $p$-vectors of re-centered bootstrap sample moments and

\footnotetext{
${ }^{5}$ In our experience, the GAUSS 9.0 quadratic programming procedure "qprog" is much faster than the Matlab 7 procedure "quadprog."

${ }^{6}$ Note that when a preliminary consistent estimator of a parameter $\tau$ appears, the bootstrap moment conditions need to be based on a bootstrap estimator of this preliminary estimator. In such cases, the
} 
$p \times p$ bootstrap weight matrices for $r=1, \ldots, R$ are defined by

$$
\begin{aligned}
& m_{n, r}^{*}(\theta)=n^{1 / 2}\left(\bar{m}_{n, r}^{*}(\theta)-\bar{m}_{n}(\theta)\right) \text { and } \\
& \widetilde{\Sigma}_{n, r}^{*}(\theta)=\widehat{\Sigma}_{n, r}^{*}(\theta)+\max \left\{\varepsilon-\operatorname{det}\left(\widehat{\Omega}_{n, r}^{*}(\theta)\right), 0\right\} \widehat{D}_{n, r}^{*}(\theta), \text { where } \varepsilon=.012, \\
& \widehat{D}_{n, r}^{*}(\theta)=\operatorname{Diag}\left(\widehat{\Sigma}_{n, r}^{*}(\theta)\right), \text { and } \widehat{\Omega}_{n, r}^{*}(\theta)=\widehat{D}_{n, r}^{*}(\theta)^{-1 / 2} \widehat{\Sigma}_{n, r}^{*}(\theta) \widehat{D}_{n, r}^{*}(\theta)^{-1 / 2}
\end{aligned}
$$

The idea behind the RMS critical value is to compute the critical value using only those moment inequalities that have a noticeable effect on the asymptotic null distribution of the test statistic. Note that moment inequalities that have large positive population means have little or no effect on the asymptotic null distribution. Our preferred RMS procedure employs element-by-element $t$-tests of the null hypothesis that the mean of $\bar{m}_{n, j}(\theta)$ is zero versus the alternative that it is positive for $j=1, \ldots, p$. The $j$-th moment inequality is selected if

$$
\frac{n^{1 / 2} \bar{m}_{n, j}(\theta)}{\widehat{\sigma}_{n, j}(\theta)} \leq \widehat{\kappa},
$$

where $\widehat{\sigma}_{n, j}^{2}(\theta)$ is the $(j, j)$ element of $\widehat{\Sigma}_{n}(\theta)$ for $j=1, \ldots, p$ and $\widehat{\kappa}$ is a data-dependent tuning parameter (defined in (2.10) below) that plays the role of a critical value in selecting the moment inequalities. Let $\widehat{p}$ denote the number of selected moment inequalities.

For $r=1, \ldots, R$, let $m_{n, r}^{*}(\theta, \widehat{p})$ denote the $\widehat{p}$-sub-vector of $m_{n, r}^{*}(\theta)$ that includes the $\widehat{p}$ selected moment inequalities. ${ }^{7,8}$ Analogously, let $\widehat{\Sigma}_{n, r}^{*}(\theta, \widehat{p})$ denote the $(\widehat{p} \times \widehat{p})$-sub-matrix of $\widehat{\Sigma}_{n, r}^{*}(\theta)$ that consists of the $\widehat{p}$ selected moment inequalities. The bootstrap quantity $c_{n}(\theta, \widehat{\kappa})$ is the $1-\alpha$ sample quantile of

$$
\left\{S_{2 A}\left(m_{n, r}^{*}(\theta, \widehat{p}), \widehat{\Sigma}_{n, r}^{*}(\theta, \widehat{p})\right): r=1, \ldots, R\right\}
$$

where $S_{2 A}(\cdot, \cdot)$ is defined as in $(2.3)$ but with $p$ replaced by $\widehat{p}$.

An "asymptotic normal" version of $c_{n}(\theta, \widehat{\kappa})$ is obtained by replacing the bootstrap quantities $m_{n, r}^{*}(\theta, \widehat{p})$ and $\widehat{\Sigma}_{n, r}^{*}(\theta, \widehat{p})$ in $(2.8)$ by $\widehat{\Sigma}_{n}^{1 / 2}(\theta, \widehat{p}) Z_{r}^{*}$ and $\widehat{\Sigma}_{n}(\theta, \widehat{p})$, respectively, where $\widehat{\Sigma}_{n}(\theta, \widehat{p})$ denotes the $(\widehat{p} \times \widehat{p})$-sub-matrix of $\widehat{\Sigma}_{n}(\theta)$ that consists of the $\widehat{p}$ selected asymptotic normal version of the critical value may be much quicker to compute.

${ }^{7}$ Note that $m_{n, r}^{*}(\theta, \widehat{p})$ depends not only on the number of moments selected, $\widehat{p}$, but which moments are selected. For simplicity, this is suppressed in the notation.

${ }^{8}$ By definition, $\widehat{p} \geq 1$, i.e., at least one moment must be selected. For specificity, $m_{n, r}^{*}(\theta, \widehat{p})$ equals the last element of $m_{n, r}^{*}(\theta)$ if no moments are selected via (2.7). 
moment inequalities, $Z_{r}^{*} \sim$ i.i.d. $N\left(0_{\widehat{p}}, I_{\widehat{p}}\right)$ for $r=1, \ldots, R$, and $\left\{Z_{r}^{*}: r=1, \ldots, R\right\}$ are independent of $\left\{W_{i}: i \leq n\right\}$ conditional on $\widehat{p}$.

The tuning parameter $\widehat{\kappa}$ in (2.7) and the size-correction factor $\widehat{\eta}$ in (2.5) depend on the estimator $\widehat{\Omega}_{n}(\theta)$ of the asymptotic correlation matrix $\Omega(\theta)$ of $n^{1 / 2} \bar{m}_{n}(\theta)$. In particular, they depend on $\widehat{\Omega}_{n}(\theta)$ through a $[-1,1]$-valued function $\delta\left(\widehat{\Omega}_{n}(\theta)\right)$ that is a measure of the amount of negative dependence in the correlation matrix $\widehat{\Omega}_{n}(\theta)$. We define

$$
\delta(\Omega)=\text { smallest off-diagonal element of } \Omega,
$$

where $\Omega$ is a $p \times p$ correlation matrix. The moment selection tuning parameter $\widehat{\kappa}$ and the size-correction factor $\widehat{\eta}$ are defined by

$$
\widehat{\kappa}=\kappa\left(\widehat{\delta}_{n}(\theta)\right) \text { and } \widehat{\eta}=\eta_{1}\left(\widehat{\delta}_{n}(\theta)\right)+\eta_{2}(p) \text {, where } \widehat{\delta}_{n}(\theta)=\delta\left(\widehat{\Omega}_{n}(\theta)\right) \text {. }
$$

Table I provides values of $\kappa(\delta), \eta_{1}(\delta)$, and $\eta_{2}(p)$ for $\delta \in[-1,1]$ and $p \in\{2,3, \ldots, 10\}$ for tests with level $\alpha=.05$ and CS's with level $1-\alpha=.95$. AJ2 provides simulated values of the mean and standard deviation of the asymptotic distribution of $c_{n}(\theta, \widehat{\kappa})$. These results, combined with the values of $\eta_{1}(\delta)$ and $\eta_{2}(p)$ in Table I, show that the size-correction factor $\widehat{\eta}$ typically is small compared to $c_{n}(\theta, \widehat{\kappa})$, but not negligible. ${ }^{9}$

Computation of the $\eta_{2}(p)$ values given in Table I by simulation is not easy because it requires computing the (asymptotic) maximum null rejection probability (MNRP) over a large number of null mean vectors $\mu$ and correlation matrices $\Omega$. For this reason, we only provide $\eta_{2}(p)$ values for $p \leq 10$. For the correlation matrices, we consider both a fixed grid and randomly generated matrices. For the null mean vectors $\mu \in R_{+, \infty}^{p}$, computation of the $\eta_{2}(p)$ values is carried out initially for mean vectors that consist only of $0^{\prime} s$ and $\infty^{\prime} s$. Then, the differences are computed between the values obtained by maximization over such $\mu$ vectors and the values obtained by maximization over $\mu$ vectors that lie in (i) a fixed full grid, (ii) two partial grids, and (iii) 1,000 or 100,000 randomly generated $\mu$ vectors (depending on the variance matrix). The differences are found to be .0000 in most cases and small $(\leq .0018)$ in all cases, see AJ2 for details. These results indicate, although do not establish unequivocally, that the maxima over $\mu \in R_{+, \infty}^{p}$ are obtained at $\mu$ vectors that consist only of $0^{\prime} s$ and $\infty^{\prime} s$.

\footnotetext{
${ }^{9}$ For example, for $p=10, \Omega=I_{10}$, five moment inequalities binding, and five moment inequalities completely slack, the mean and standard deviation of the asymptotic distribution of $c_{n}(\theta, \widehat{\kappa})$ are 7.2 and .57 , respectively, whereas the size-correction factor is .614.
} 
In sum, the preferred RMS critical value, $c_{n}(\theta)$, and CS are computed using the following steps. One computes (i) $\widehat{\Omega}_{n}(\theta)$ defined in $(2.4)$, (ii) $\widehat{\delta}_{n}(\theta)=$ smallest off-diagonal element of $\widehat{\Omega}_{n}(\theta)$, (iii) $\widehat{\kappa}=\kappa\left(\widehat{\delta}_{n}(\theta)\right)$ using Table I, (iv) $\widehat{\eta}=\eta_{1}\left(\widehat{\delta}_{n}(\theta)\right)+\eta_{2}(p)$ using Table I, (v) the vector of selected moments using (2.7), (vi) the selected bootstrap sample moments, correlation matrices, and weight matrices $\left\{\left(m_{n, r}^{*}(\theta, \widehat{p}), \widehat{\Sigma}_{n, r}^{*}(\theta, \widehat{p}), \widetilde{\Sigma}_{n, r}^{*}(\theta, \widehat{p})\right)\right.$ : $r=1, \ldots, R\}$, defined in (2.6) with the non-selected moment inequalities omitted, (vii) $c_{n}(\theta, \widehat{\kappa})$, which is the .95 sample quantile of $\left\{S_{2 A}\left(m_{n, r}^{*}(\theta, \widehat{p}), \widehat{\Sigma}_{n, r}^{*}(\theta, \widehat{p})\right): r=1, \ldots, R\right\}$ (for a test of level .05 and a CS of level .95) and (viii) $c_{n}(\theta)=c_{n}(\theta, \widehat{\kappa})+\widehat{\eta}$. The preferred RMS confidence set is computed by determining all the values $\theta$ for which the null hypothesis that $\theta$ is the true value is not rejected. For the asymptotic normal version of the recommended RMS critical value, in step (vi) one computes the selected sub-vector and sub-matrix of $\widehat{\Sigma}_{n}^{1 / 2}(\theta, \widehat{p}) Z_{r}^{*}$ and $\widehat{\Sigma}_{n}(\theta, \widehat{p})$, defined in the paragraph following (2.8), and in step (vii) one computes the .95 sample quantile with these quantities in place of $m_{n, r}^{*}(\theta, \widehat{p})$ and $\widehat{\Sigma}_{n, r}^{*}(\theta, \widehat{p})$, respectively.

To compute the recommended bootstrap RMS test using $R=10,000$ simulation repetitions takes $1.3,1.5$, and 2.7 seconds when $p=2,4$, and 10, respectively, and $n=250$ using GAUSS on a PC with a $3.4 \mathrm{GHz}$ processor. For the "asymptotic normal" version, the times are $.20, .25$, and .45 seconds.

When constructing a CS, if the computation time is burdensome (because one needs to carry out many tests with different values of $\theta$ as the null value), then a useful approach is to map out the general features of the CS using the "asymptotic normal" version of the $\mathrm{MMM} / t$-Test $/ \kappa=2.35$ test, which is extremely fast to compute, and then switch to the bootstrap version of the recommended RMS test to find the boundaries of the CS more precisely. ${ }^{10}$

\footnotetext{
${ }^{10}$ The "asymptotic normal" version of the $\mathrm{MMM} / t$-Test $/ \kappa=2.35$ test is defined just as the recommended RMS test is defined but with $\left(S_{1}, \kappa=2.35, \eta=0\right)$ in place of $\left(S_{2 A}, \widehat{\kappa}, \widehat{\eta}\right)$, respectively, where $S_{1}$ is defined in (3.2), and with the bootstrap replaced by the normal asymptotic distribution. The bootstrap version of this test is much slower to compute than the asymptotic normal version and, hence, we do not recommend that it is used for this purpose. The computation times for the "asymptotic normal" version of the $\mathrm{MMM} / t$-Test $/ \kappa=2.35$ test are $.007, .014$, and .03 seconds when $p=2,4$, and 10, respectively.
} 


\section{Test Statistics and Critical Values}

We now describe the justification for the recommended RMS test. Details are given in AJ2. The test statistics $T_{n}(\theta)$ that we consider are of the form

$$
T_{n}(\theta)=S\left(n^{1 / 2} \bar{m}_{n}(\theta), \widehat{\Sigma}_{n}(\theta)\right)
$$

where $S$ is a real function on $(R \cup\{+\infty\})^{p} \times \mathcal{V}$ and $\mathcal{V}$ is the space of $p \times p$ variance matrices. The leading examples of $S$ are the AQLR function $S_{2 A}$ defined above, the QLR function $S_{2}$, which is the same as $S_{2 A}$ in $(2.3)$ but with $\varepsilon=0$ (and hence $\widetilde{\Sigma}_{n}(\theta)=\widehat{\Sigma}_{n}(\theta)$ ), the modified method of moments (MMM) function $S_{1}$, and the SumMax function $S_{3}$ :

$$
S_{1}(m, \Sigma)=\sum_{j=1}^{p}\left[m_{j} / \sigma_{j}\right]_{-}^{2} \text { and } S_{3}(m, \Sigma)=\sum_{j=1}^{p_{1}}\left[m_{(j)} / \sigma_{(j)}\right]_{-}^{2}
$$

where $[x]_{-}=\min \{x, 0\}, m=\left(m_{1}, \ldots, m_{p}\right)^{\prime}, \sigma_{j}^{2}$ is the $j$ th diagonal element of $\Sigma$, $\left[m_{(j)} / \sigma_{(j)}\right]_{-}^{2}$ denotes the $j$ th largest value among $\left\{\left[m_{\ell} / \sigma_{\ell}\right]_{-}^{2}: \ell=1, \ldots, p\right\}$, and $p_{1}<p$ is some specified integer. ${ }^{11,12,13}$ The MMM statistic $S_{1}$ has been used by Pakes, Porter, Ho, and Ishii (2004), CHT, Fan and Park (2007), Romano and Shaikh (2008), AG, AS, and Bugni (2010); the (unadjusted) QLR statistic has been used by AG, AS, and Rosen (2008); and the Max and SumMax statistics $S_{3}$ have been used by AG, AS, and Azeem Shaikh. ${ }^{14}$

We consider the class of GMS critical values discussed in AS. They rely on a tuning parameter $\kappa$ and moment selection functions $\varphi_{j}:(R \cup\{+\infty\})^{p} \times \Psi \rightarrow R_{+}$for $j \leq p$, where $\Psi$ is the set of all $p \times p$ correlation matrices. The leading examples of $\varphi_{j}$ are

$$
\begin{aligned}
& \varphi_{j}^{(1)}(\xi, \Omega)=\left\{\begin{array}{ll}
0 & \text { if } \xi_{j} \leq 1 \\
\infty & \text { if } \xi_{j}>1,
\end{array} \varphi_{j}^{(2)}(\xi, \Omega)=\left[\kappa\left(\xi_{j}-1\right)\right]_{+}, \varphi_{j}^{(3)}(\xi, \Omega)=\left[\xi_{j}\right]_{+},\right. \\
& \varphi_{j}^{(4)}(\xi, \Omega)=\kappa \xi_{j} 1\left(\xi_{j}>1\right), \text { and } \varphi_{j}^{(0)}(\xi, \Omega)=0
\end{aligned}
$$

for $j \leq p$, where $[x]_{+}=\max \{x, 0\}, \xi=\left(\xi_{1}, \ldots, \xi_{p}\right)^{\prime}, \Omega$ is a $p \times p$ correlation matrix,

\footnotetext{
${ }^{11}$ When constructing a CS, a natural choice for $p_{1}$ is the dimension $d$ of $\theta$, see below.

${ }^{12} \mathrm{With}$ the functions $S_{1}, S_{2 A}$, and $S_{3}$, there is no restriction on the parameter space for the variance matrix $\Sigma$ of the moment conditions $-\Sigma$ can be singular.

${ }^{13}$ Several papers in the literature use a variant of $S_{1}$ that is not invariant to rescaling of the moment functions (i.e., with $\sigma_{j}=1$ for all $j$ ). This is not desirable in terms of the power of the resulting test.

${ }^{14}$ Personal communication.
} 
and $\kappa$ in $\varphi_{j}^{(2)}$ and $\varphi_{j}^{(4)}$ is the tuning parameter $\kappa$. Let $\varphi(\xi, \Omega)=\left(\varphi_{1}(\xi, \Omega), \ldots, \varphi_{p}(\xi, \Omega)\right)^{\prime}$ (for any $\varphi_{j}(\xi, \Omega)$ as in (3.3)). CHT, AS, and Bugni (2010) consider the function $\varphi^{(1)}$; Canay (2010) considers $\varphi^{(2)}$; AS considers $\varphi^{(3)}$; and Fan and Park (2007) use a nonscale-invariant version of $\varphi^{(4)}$. The function $\varphi^{(1)}$ generates the recommended "moment selection $t$-test" procedure of (2.7), see AJ2 for details. The function $\varphi^{(0)}$ generates a critical value based on the least-favorable distribution evaluated at an estimator of the true variance matrix $\Sigma$. It only depends on the data through the estimation of $\Sigma$. It is referred to as the "plug-in" asymptotic (PA) critical value. (No value $\kappa$ is needed for this critical value.) Another $\varphi$ function is the modified moment selection criterion (MMSC) $\varphi^{(5)}$ function introduced in AS. It is computationally more expensive than the functions $\varphi^{(1)}-\varphi^{(4)}$ considered above, but uses all of the information in the $p$-vector of moment conditions to decide which moments to select. It is a one-sided version of the information-criterion-based moment selection criterion considered in Andrews (1999). For brevity, we do not define $\varphi^{(5)}$ here, but we consider it below.

For a GMS critical value as in AS, $\left\{\kappa=\kappa_{n}: n \geq 1\right\}$ is a sequence of constants that diverges to infinity as $n \rightarrow \infty$, such as $\kappa_{n}=(\ln n)^{1 / 2}$. In contrast, for an RMS critical value, $\widehat{\kappa}$ does not go to infinity as $n \rightarrow \infty$ and is data-dependent. Data-dependence of $\widehat{\kappa}$ is obtained by taking $\widehat{\kappa}$ to depend on $\widehat{\Omega}_{n}(\theta)$ : $\widehat{\kappa}=\kappa\left(\widehat{\Omega}_{n}(\theta)\right)$, where $\kappa(\cdot)$ is an $R_{++}$-valued function. We justify RMS critical values using asymptotics in which $\kappa$ equals a finite constant plus $o_{p}(1)$, rather than asymptotics in which $\kappa \rightarrow \infty$ as $n \rightarrow \infty$. This differs from the asymptotics in other papers in the moment inequality literature.

There are four reasons for using finite- $\kappa$ asymptotics. First, they provide better approximations because $\kappa$ is finite, not infinite, in any given application. Second, for any given $(S, \varphi)$, they allow one to compute a best $\kappa$ value in terms of asymptotic average power, which in turn allows one to compare different $(S, \varphi)$ functions (each evaluated at its own best $\kappa$ value) in terms of asymptotic average power. One cannot determine a best $\kappa$ value in terms of asymptotic average power when $\kappa \rightarrow \infty$ because asymptotic power is always higher if $\kappa$ is smaller, asymptotic size does not depend on $\kappa$, and finite-sample size is worse if $\kappa$ smaller. $^{15}$ Third, for the recommended $(S, \varphi)$ functions, the finite- $\kappa$ asymptotic formula for the best $\kappa$ value lets one determine a data-dependent $\kappa$ value that is approximately optimal in terms of asymptotic average power. Fourth, finite- $\kappa$

\footnotetext{
${ }^{15}$ This does not imply that one cannot size-correct a test and then consider the $\kappa \rightarrow \infty$ asymptotic properties of such a test. Rather, the point is that $\kappa \rightarrow \infty$ asymptotics do not allow one to determine a suitable formula for size correction for the reason given.
} 
asymptotics permit one to compute size-correction factors that depend on $\kappa$, which is a primary determinant of a test's finite-sample size. In contrast, if $\kappa \rightarrow \infty$ the asymptotic properties of tests under the null hypothesis do not depend on $\kappa$. Even the higher-order errors in null rejection probabilities do not depend on $\kappa$, see Bugni (2010). Thus, with $\kappa \rightarrow \infty$ asymptotics, the determination of a desirable size-correction factor based on $\kappa$ is not possible.

For brevity, the finite- $\kappa$ asymptotic results are given in AJ2. These results include uniform asymptotic size and $n^{-1 / 2}$-local power results. We use these results to compare different $(S, \varphi)$ functions below and to develop recommended $\widehat{\kappa}$ and $\widehat{\eta}$ values.

For $Z^{*} \sim N\left(0_{p}, I_{p}\right)$ and $\beta \in(R \cup\{+\infty\})^{p}$, let $q_{S}(\beta, \Omega)$ denote the $1-\alpha$ quantile of $S\left(\Omega^{1 / 2} Z^{*}+\beta, \Omega\right)$. For constants $\kappa>0$ and $\eta \geq 0$, define

$$
\begin{aligned}
& \operatorname{AsyPow}(\mu, \Omega, S, \varphi, \kappa, \eta) \\
= & P\left(S\left(\Omega^{1 / 2} Z^{*}+\mu, \Omega\right)>q_{S}\left(\varphi\left(\kappa^{-1}\left[\Omega^{1 / 2} Z^{*}+\mu\right], \Omega\right), \Omega\right)+\eta\right),
\end{aligned}
$$

where $\mu \in R^{p}$ and $\Omega \in \Psi$. The asymptotic power of an RMS test of the null hypothesis that the true value is $\theta$, based on $(S, \varphi)$ with data-dependent $\widehat{\kappa}=\kappa\left(\widehat{\Omega}_{n}(\theta)\right)$, and $\widehat{\eta}=$ $\eta\left(\widehat{\Omega}_{n}(\theta)\right)$, is shown in AJ2 to be AsyPow $(\mu, \Omega(\theta), S, \varphi, \kappa(\Omega(\theta)), \eta(\Omega(\theta)))$, where $\mu$ is a $p$-vector whose elements depend on the limits (as $n \rightarrow \infty)$ of the normalized population means of the $p$ moment inequalities and $\Omega(\theta)$ is the population correlation matrix of the moment functions evaluated at the null value $\theta$.

We compare the power of different RMS tests by comparing their asymptotic average power for a chosen set $\mathcal{M}_{p}(\Omega)$ of alternative parameter vectors $\mu \in R^{p}$ for a given correlation matrix $\Omega$. The asymptotic average power of the RMS test based on $(S, \varphi, \kappa, \eta)$ for constants $\kappa>0$ and $\eta \geq 0$ is

$$
\left|\mathcal{M}_{p}(\Omega)\right|^{-1} \sum_{\mu \in \mathcal{M}_{p}(\Omega)} \operatorname{AsyPow}(\mu, \Omega, S, \varphi, \kappa, \eta)
$$

where $\left|\mathcal{M}_{p}(\Omega)\right|$ denotes the number of elements in $\mathcal{M}_{p}(\Omega)$.

We are interested in constructing tests that yield CS's that are as small as possible. The boundary of a CS, like the boundary of the identified set, is determined at any given point by the moment inequalities that are binding at that point. The number of binding moment inequalities at a point depends on the dimension, $d$, of the parameter $\theta$. Typically, the boundary of a confidence set is determined by $d$ (or fewer) moment 
inequalities. That is, at most $d$ moment inequalities are binding and at least $p-d$ are slack. In consequence, we specify the sets $\mathcal{M}_{p}(\Omega)$ considered below to be ones for which most vectors $\mu$ have half or more elements positive (since positive elements correspond to non-binding inequalities), which is suitable for the typical case in which $p \geq 2 d$.

To compare $(S, \varphi)$ functions based on asymptotic $\mathcal{M}_{p}(\Omega)$-average power requires choices of functions $(\kappa(\cdot), \eta(\cdot))$. We use the functions $\kappa^{*}(\Omega)$ and $\eta^{*}(\Omega)$ that are optimal in terms of maximizing asymptotic $\mathcal{M}_{p}(\Omega)$-average power. These are determined numerically, see AJ2 for details. Given $\Omega, \kappa^{*}(\Omega)$, and $\eta^{*}(\Omega)$, we compare $(S, \varphi)$ functions by comparing their values of the quantity in (3.5) evaluated at $\kappa=\kappa^{*}(\Omega)$, and $\eta=\eta^{*}(\Omega)$.

Once we have determined a recommended $(S, \varphi)$, we determine data-dependent values $\widehat{\kappa}$ and $\widehat{\eta}$ that are suitable for use with this $(S, \varphi)$ combination.

Note that generalized empirical likelihood (GEL) test statistics, including the empirical likelihood ratio (ELR) statistic, behave the same asymptotically (to the first order) as the (unadjusted) QLR statistic $T_{n}(\theta)$ based on $S_{2}$ under the null and local alternative hypotheses for nonsingular correlation matrices of the moment conditions. See Sections 8.1 and 10.3 of AG, Section 10.1 of AS, and Canay (2010). In consequence, although GEL statistics are not of the form given in (3.1), the asymptotic results of the present paper, given in AJ2, hold for such statistics under the assumptions given in AG for classes of moment condition correlation matrices whose determinants are bounded away from zero. Hence, in the latter case, the recommended $\widehat{\kappa}$ and $\widehat{\eta}$ values given in Table I can be used with GEL statistics. However, an advantage of the AQLR statistic in comparison to GEL statistics is that its asymptotic properties are known and well-behaved whether or not the moment condition correlation matrix is singular. There are also substantial computational reasons to prefer the AQLR statistic to GEL statistics such as ELR, see Section 6 below.

\section{Asymptotic Average Power Comparisons}

In the numerical work reported here, we focus on results for $p=2,4$, and 10. For each value of $p$, we consider three correlation matrices $\Omega$ : $\Omega_{N e g}, \Omega_{Z \text { ero }}$, and $\Omega_{\text {Pos }}$. The matrix $\Omega_{Z \text { ero }}$ equals $I_{p}$ for $p=2,4$, and 10 . The matrices $\Omega_{N e g}$ and $\Omega_{P o s}$ are Toeplitz matrices with correlations on the diagonals (as they go away from the main diagonal) given by the following: For $p=2: \rho=-.9$ for $\Omega_{N e g}$ and $\rho=.5$ for $\Omega_{P o s}$. For $p=4: \rho=(-.9, .7,-.5)$ for $\Omega_{N e g}$ and $\rho=(.9, .7, .5)$ for $\Omega_{P o s}$. For $p=10: \rho=(-.9, .8,-.7, .6,-.5, .4,-.3, .2,-.1)$ 
for $\Omega_{N e g}$ and $\rho=(.9, .8, .7, .6, .5, \ldots, .5)$ for $\Omega_{\text {Pos }}$.

For $p=2$, the set of $\mu$ vectors $\mathcal{M}_{2}(\Omega)$ for which asymptotic average power is computed includes seven elements: $\mathcal{M}_{2}(\Omega)=\left\{\left(-\mu_{1}, 0\right),\left(-\mu_{2}, 1\right),\left(-\mu_{3}, 2\right),\left(-\mu_{4}, 3\right)\right.$, $\left.\left(-\mu_{5}, 4\right),\left(-\mu_{6}, 7\right),\left(-\mu_{7},-\mu_{7}\right)\right\}$, where $\mu_{j}$ depends on $\Omega$ and is such that the power envelope is .75 at each element of $\mathcal{M}_{2}(\Omega)$. Consistent with the discussion in Section 3, most elements of $\mathcal{M}_{2}(\Omega)$ have less than two negative elements. The positive elements of the $\mu$ vectors are chosen to cover a reasonable range of the parameter space. For brevity, the values of $\mu_{j}$ in $\mathcal{M}_{2}(\Omega)$ and the sets $\mathcal{M}_{p}(\Omega)$ for $p=4,10$ are given in AJ2. The elements of $\mathcal{M}_{p}(\Omega)$ for $p=4,10$ are selected such that the power envelope is .80 and .85, respectively, at each element of the set.

In AJ2 we also provide results for two singular $\Omega$ matrices and 19 nonsingular $\Omega$ matrices (for each $p$ ) that cover a grid of $\delta(\Omega)$ values from -1.0 to 1.0. The qualitative results reported here are found to apply as well to the broader range of $\Omega$ matrices. Some special features of the results based on the singular variance matrices are commented on below.

We compare tests based on the following functions: $(S, \varphi)=(\mathrm{MMM}, \mathrm{PA}),(\mathrm{MMM}$, t-Test), (Max, PA), (Max, t-Test), (SumMax, PA), (SumMax, t-Test), (AQLR, PA), (AQLR, t-Test), (AQLR, $\left.\varphi^{(3)}\right)$, (AQLR, $\left.\varphi^{(4)}\right)$, and (AQLR, MMSC). ${ }^{16}$ We also consider the "pure ELR" test, for which Canay (2010) establishes a large deviation asymptotic optimality result. This test rejects the null when the ELR statistic exceeds a fixed constant (that is the same for all $\Omega$ ). ${ }^{17}$ The reason for reporting results for this test is to show that these asymptotic optimality results do not provide theoretical grounds for favoring the ELR test or ELR test statistic over other tests or test statistics.

For each test, Table II reports the asymptotic average power given the $\kappa$ value that maximizes asymptotic average power for the test, denoted $\kappa=$ Best. The best $\kappa$ values are determined numerically using grid search, see AJ2 for details. For all tests and $p=2,4,10$, the best $\kappa$ values are decreasing from $\Omega_{N e g}$ to $\Omega_{Z \text { ero }}$ to $\Omega_{\text {Pos }}$. For example,

\footnotetext{
${ }^{16}$ The statistics MMM, AQLR, Max, and SumMax use the functions $S_{1}, S_{2}, S_{3}$ with $p_{1}=1$, and $S_{3}$ with $p_{1}=2$, respectively. The PA, $t$-Test, and MMSC critical values use the functions $\varphi^{(0)}, \varphi^{(1)}$, and $\varphi^{(5)}$, respectively.

${ }^{17}$ The level .05 pure ELR asymptotic critical value is determined numerically by calculating the constant for which the maximum asymptotic null rejection probability of the ELR statistic over all mean vectors in the null hypothesis and over all positive definite correlation matrices $\Omega$ is .05. See AJ2 for details. The critical values are found to be 5.07, 7.99, and 16.2 for $p=2,4$, and 10, respectively. These critical values yield asymptotic null rejection probabilities of .05 when $\Omega$ contains elements that are close to -1.0 .
} 
for the AQLR/t-Test test, the best $\kappa$ values for $\left(\Omega_{N e g}, \Omega_{Z \text { ero }}, \Omega_{P o s}\right)$ are $(2.5,1.4, .6)$ for $p=10,(2.5,1.4, .8)$ for $p=4$, and $(2.6,1.7, .6)$ for $p=2$.

The asymptotic power results are size-corrected. ${ }^{18,19}$ The critical values, size-correction factors, and power results are each calculated using 40, 000 simulation repetitions, except where stated otherwise, which yields a simulation standard error of .0011 for the power results.

Table II shows that the MMM/PA test has very low asymptotic power compared to the AQLR/t-Test/ $\kappa$ Best test (which is shown in boldface) especially for $p=4,10$. Similarly, the Max/PA and SumMax/PA tests have low power. The AQLR/PA test has better power than the other PA tests, but it is still very low compared to the AQLR/tTest $/ \kappa$ Best test.

Table II also shows that the MMM/t-Test/ $\kappa$ Best test has equal asymptotic average power to the AQLR/t-Test/ $/ \kappa$ Best test for $\Omega_{\text {Zero }}$ and only slightly lower power for $\Omega_{\text {Pos }}$. But, it has substantially lower power for $\Omega_{N e g}$. For example, for $p=10$, the comparison is .18 versus .55. The Max $/ t$-Test $/ \kappa$ Best test has noticeably lower average power than the AQLR/t-Test/ $/ \kappa$ Best test for $\Omega_{N e g}$, slightly lower power for $\Omega_{Z \text { ero }}$, and essentially equal power for $\Omega_{P o s}$. It is strongly dominated in terms of average power. The SumMax/t-Test/ $/$ Best test also is strongly dominated by the AQLR/ $t$-Test $/ \kappa$ Best test in terms of asymptotic average power. The power differences between these two tests are especially large for $\Omega_{N e g}$. For example, for $p=10$ and $\Omega_{N e g}$, their powers are .20 and .55 , respectively.

Next we compare tests that use the AQLR test statistic but different critical valuesdue to the use of different functions $\varphi$. The AQLR/ $\varphi^{(2)} / \kappa$ Best test is essentially dominated by the AQLR/t-Test/ $/$ Best, although the differences are not large. The AQLR $/ \varphi^{(3)} / \kappa$ Best test has noticeably lower asymptotic average power than the AQLR/tTest/ $\kappa$ Best test for $\Omega_{N e g}$, somewhat lower power for $\Omega_{Z \text { ero }}$, and equal power for $\Omega_{\text {Pos }}$. The differences increase with $p$.

The AQLR $/ \varphi^{(4)} / \kappa$ Best test has almost the same asymptotic average power as the AQLR $/ t$-Test $/ \kappa$ Best test for $\Omega_{Z \text { ero }}$ and $\Omega_{\text {Pos }}$ and slightly lower power for $\Omega_{N e g}$. This

\footnotetext{
${ }^{18}$ Size-correction here is done for the fixed known value of $\Omega$. It is not based on the least-favorable $\Omega$ matrix because the results are asymptotic and $\Omega$ can be estimated consistently.

${ }^{19}$ The maximum null rejection probability calculations used in the size correction were calculated using $\mu$ vectors that consist of $0^{\prime} s$ and $\infty^{\prime} s$. Then, additional calculations were carried out to determine whether the maximum over $\mu \in R_{+, \infty}^{p}$ is attained at such a $\mu$ vector in each case. No evidence was found to suggest otherwise. See Section 7 of the Supplemental Material for details.
} 
is because the $\varphi^{(4)}$ and $\varphi^{(1)}$ functions are similar. The AQLR/MMSC/ $\kappa$ Best test and AQLR/t-Test/ $\kappa$ Best tests have quite similar power. Nevertheless, the AQLR/MMSC/ $\kappa$ Best test is not the recommended test for reasons given below. We experimented with several smooth versions of the $\varphi^{(1)}$ critical value function in conjunction with the AQLR statistic. We were not able to find any that improved upon the asymptotic average power of the AQLR/t-Test/ $\kappa$ Best test. Some were inferior. All such tests have substantial disadvantages relative to the AQLR/t-test in terms of the computational ease of determining suitable data-dependent $\kappa$ and $\eta$ values.

In conclusion, we find that the best $(S, \varphi)$ choices in terms of asymptotic average power (based on $\kappa=$ Best) are: AQLR/t-Test and AQLR/MMSC, followed closely by $\mathrm{AQLR} / \varphi^{(2)}$ and AQLR/ $\varphi^{(4)}$. Each of these tests out-performs the PA tests by a wide margin in terms of asymptotic power.

The AQLR/MMSC test has the following drawbacks: (i) its computation time is very high when $p$ is large, such as $p=10$, because the test statistic must be computed for all $2^{p}$ possible combinations of selected moment vectors and (ii) the best $\kappa$ value varies widely with $\Omega$ and $p$, which makes it quite difficult to specify a data-dependent $\kappa$ value that performs well. Similarly, the $\mathrm{AQLR} / \varphi^{(2)}$ and $\mathrm{AQLR} / \varphi^{(4)}$ tests have substantial computational drawbacks for determining a data-dependent $\kappa$ values, see AJ2 for details.

Based on the power results discussed above and on the computational factors, we take the AQLR/t-Test to be the recommended test and we develop data-dependent $\widehat{\kappa}$ and $\widehat{\eta}$ for this test.

The last row of Table II gives the asymptotic power envelope, which is a "unidirectional" envelope, see AJ2 for details. One does not expect a test that is designed to perform well for multi-directional alternatives to be on, or close to, the uni-directional envelope. In fact, it is surprising how close the AQLR/t-Test/ $/$ Best test is to the power envelope when $\Omega=\Omega_{\text {Pos }}$. As expected, the larger is $p$ the greater is the difference between the power of a test designed for $p$-directional alternatives and the uni-directional power envelope.

When the sample correlation matrix is singular, the QLR test statistic can be defined using the Moore-Penrose generalized inverse in the definition of the weighting matrix. Let MP-QLR denote this statistic. For the case of singular correlation matrices, AJ2 provides asymptotic power comparisons of the AQLR/t-Test/ $/$ Best test, the MP-QLR/tTest $/ \kappa$ Best test, and several other tests.

The results show that the AQLR/t-Test/ $/$ Best test has vastly superior asymptotic 
average power to that of the MP-QLR/t-Test/ $\kappa$ Best test (e.g., .98 versus .29 when $p=10$ ) when the correlation matrix exhibits perfect negative correlation and the same power when only perfect positive correlation is present. Hence, it is clear that the adjustment made to the QLR statistic is beneficial. The results also show that the AQLR/t-Test/ $\kappa$ Best test strongly dominates tests based on the MMM and Max statistics in terms of asymptotic average power with singular correlation matrices.

Finally, results for the "pure ELR" test show that it has very poor asymptotic power properties. ${ }^{20}$ For example, for $p=10$, its power ranges $1 / 3$ to $1 / 7$ that of the AQLR/tTest/ $\kappa$ Best test (and of the feasible AQLR/t-Test/ $\kappa$ Auto test, which is the recommended test of Section 2). The poor power properties of this "asymptotically optimal" test imply that the (generalized Neyman-Pearson) large deviations asymptotic optimality criterion is not a suitable criterion in this context. ${ }^{21}$

Note that the poor power of the "pure ELR" test does not imply that the ELR test statistic is a poor choice of test statistic. When combined with a good critical value, such as the data-dependent critical value recommended in this paper or a similar critical value, it yields a test with very good power. The point is that the large deviations asymptotic optimality result does not provide convincing evidence in favor of the ELR statistic.

\section{Approximately Optimal $\kappa(\Omega)$ and $\eta(\Omega)$ Functions}

Next, we describe how the recommended $\kappa(\Omega)$ and $\eta(\Omega)$ functions for the AQLR/tTest test, defined in Section 2 and referred to, are determined.

First, for $p=2$ and given $\rho \in(-1,1)$, where $\rho$ denotes the correlation that appears in $\Omega$, we compute numerically the values of $\kappa$ that maximize the asymptotic average (size-corrected) power of the nominal .05 AQLR/t-Test test over a fine grid of $31 \kappa$ values. We do this for each $\rho$ in a fine grid of 43 values. Because the power results

\footnotetext{
${ }^{20}$ The power of the pure ELR test and AQLR/t-Test/ $\kappa$ Auto test, which is the recommended test of Section 2 , in the nine cases considered in Table II are: for $p=10,(.19, .55),(.17, .67)$, and $(.12, .82)$; for $p=4,(.44, .59),(.42, .69),(.39, .78)$; and for $p=2,(.57, .65),(.55, .69)$, and $(.54, .73)$. See Table S-XIII of AJ2.

${ }^{21}$ In our view, the large-deviation asymptotic optimality criterion is not appropriate when comparing tests with substantially different asymptotic properties under non-large deviations. In particular, this criterion is questionable when the alternative hypothesis is multi-dimensional because it implies that a test can be "optimal" against alternatives in all directions, which is incompatible with the finite sample and local asymptotic behavior of tests in most contexts.
} 
are size-corrected, a by-product of determining the best $\kappa$ value for each $\rho$ value is the size-correction value $\eta$ that yields asymptotically correct size for each $\rho$.

Second, by a combination of intuition and the analysis of numerical results, we postulate that for $p \geq 3$ the optimal function $\kappa^{*}(\Omega)$ is well approximated by a function that depends on $\Omega$ only through the $[-1,1]$-valued function $\delta(\Omega)$ defined in $(2.9)$.

The explanation for this is as follows: (i) Given $\Omega$, the value $\kappa^{*}(\Omega)$ that yields maximum asymptotic average power is such that the size-correction value $\eta^{*}(\Omega)$ is not very large. (This is established numerically for a variety of $p$ and $\Omega$.) The reason is that the larger is $\eta^{*}(\Omega)$, the larger is the fraction, $\eta^{*}(\Omega) /\left(c_{n}\left(\theta, \kappa^{*}(\Omega)\right)+\eta^{*}(\Omega)\right)$ of the critical value that does not depend on the data (for $\Omega$ known), the closer is the critical value to the PA critical value that does not depend on the data at all (for known $\Omega$ ), and the lower is the power of the test for $\mu$ vectors that have less than $p$ elements negative and some elements strictly positive. (ii) The size-correction value $\eta^{*}(\Omega)$ is small if the rejection probability at the least-favorable null vector $\mu$ is close to $\alpha$ when using the sizecorrection factor $\eta(\Omega)=0$. (This is self-evident.) (iii) We postulate that null vectors $\mu$ that have two elements equal to zero and the rest equal to infinity are nearly leastfavorable null vectors. ${ }^{22}$ If true, then the size of the AQLR/t-Test test depends on the two-dimensional sub-matrices of $\Omega$ that are the correlation matrices for the cases where only two moment conditions appear. (iv) The size of a test for given $\kappa$ and $p=2$ is decreasing in the correlation $\rho$. In consequence, the least-favorable two-dimensional submatrix of $\Omega$ is the one with the smallest correlation. Hence, the value of $\kappa$ that makes the size of the test equal to $\alpha$ for a small value of $\eta$ is (approximately) a function of $\Omega$ through $\delta(\Omega)$ defined in $(2.9)$. (Note that this is just a heuristic explanation. It is not intended to be a proof.)

Next, because $\delta(\Omega)$ corresponds to a particular $2 \times 2$ submatrix of $\Omega$ with correlation $\delta(=\delta(\Omega)$ ), we take $\kappa(\Omega)$ to be the value that maximizes asymptotic average power when $p=2$ and $\rho=\delta$, as specified in Table I and described in the second paragraph of this section. We take $\eta(\Omega)$ to be the value determined by $p=2$ and $\delta$, i.e., $\eta_{1}(\delta)$ in $(2.10)$ and Table I, but allow for an adjustment that depends on $p$, viz., $\eta_{2}(p)$, that is defined to guarantee that the test has correct asymptotic significance level (up to numerical error).

\footnotetext{
${ }^{22}$ The reason for this postulation is that a test with given $\kappa$ has larger null rejection probability the more negative are the correlations between the moments. A variance matrix of dimension three by three or greater has restrictions on its correlations imposed by the positive semi-definiteness property. If all of the correlations are equal, they cannot be arbitarily close to -1 . In constrast, with a two-dimensional variance matrix, the correlation can be arbitarily close to -1 .
} 
See AJ2 for details.

We refer to the proposed method of selecting $\kappa(\Omega)$ and $\eta(\Omega)$, described in Section 2 , as the $\kappa$ Auto method. We examine numerically how well the $\kappa$ Auto method does in approximating the best $\kappa$, viz., $\kappa^{*}(\Omega) .{ }^{23}$ We provide four groups of results and consider $p=2,4,10$ for each group. The first group consists of the three $\Omega$ matrices considered in Table II. The rows of Table II for the AQLR/t-Test/ $\kappa$ Best and AQLR/ $t$-Test/ $/$ Auto tests show that the $\kappa$ Auto method works very well. It has the same asymptotic average power as the AQLR/t-Test/ $/$ Best test for all $p$ and $\Omega$ values except one case where the difference is just .01.

The second group consists of a set of $19 \Omega$ matrices for which $\delta(\Omega)$ takes values on a grid in $[-.99, .99]$. In 53 of the $57(=3 \times 19)$ cases, the difference in asymptotic average power of the AQLR/t-Test/ $\kappa$ Best and AQLR/t-Test/ $\kappa$ Auto tests is less than .01 .

The third group consists of two singular $\Omega$ matrices. One with perfect negative and positive correlations and the other with perfect positive correlations. The AQLR/tTest/ $\kappa$ Auto test has the same asymptotic average power as the AQLR/t-Test/ $/$ Best test for $3(p, \Omega)$ combinations, power that is lower by .01 for 2 combinations, and power that is lower by .02 for one combination.

The fourth group consists 500 randomly generated $\Omega$ matrices for $p=2,4$ and 250 randomly generated $\Omega$ matrices for $p=10$. For $p=2$, across the $500 \Omega$ matrices, the asymptotic average power differences have average equal to .0010, standard deviation equal to .0032 , and range equal to $[.000, .022]$. For $p=4$, across the $500 \Omega$ matrices, the average power difference is .0012, the standard deviation is .0016, and the range is $[.000, .010]$. For $p=10$, across the $250 \Omega$ matrices, the average power differences have average equal to .0183, standard deviation equal to .0069, and range equal to [.000,.037].

In conclusion, the $\kappa$ Auto method performs very well in terms of selecting $\kappa$ values that maximize the asymptotic average power.

\section{Finite-Sample Results}

The recommended RMS test, AQLR/t-Test/ $\kappa$ Auto, can be implemented in finite samples via the "asymptotic normal" and the bootstrap versions of the $t$-Test/ $\kappa$ Auto critical value. Here we determine which of these two methods performs better in finite samples. We also compare these tests to the bootstrap version of the ELR/ $t$-Test/ $\kappa$ Auto

\footnotetext{
${ }^{23}$ For brevity, details of the numerical results are given in AJ2.
} 
test, which has the same first-order asymptotic properties as the AQLR-based tests (for correlation matrices whose determinants are bounded away from zero by $\varepsilon=.012$ or more). See Sec. 6.3 .3 of AJ2 for the definition of the ELR statistic and details of its computation.

In short, we find that the bootstrap version (denoted Bt in Table III) of the AQLR/tTest/ $\kappa$ Auto test performs better than the asymptotic normal version (denoted Nm) in terms of the closeness of its null rejection probabilities to its nominal level and similarly on average in terms of its power. The AQLR bootstrap test also performs slightly better than the ELR bootstrap test in terms of power, is noticeably superior in terms of computation time, and is essentially the same (up to simulation error) in terms of null rejection probabilities. In addition, the AQLR bootstrap test is found to perform quite well in an absolute sense. Its null rejection probabilities are close to its nominal level and the difference between its finite-sample and asymptotic power is relatively small.

We provide results for sample size $n=100$. We consider the same correlation matrices $\Omega_{N e g}, \Omega_{Z \text { ero }}$, and $\Omega_{\text {Pos }}$ as above and the same numbers of moment inequalities $p=2,4$, and 10 . We take the mean zero variance $I_{p}$ random vector $Z^{\dagger}=$ $\operatorname{Var}^{-1 / 2}\left(m\left(W_{i}, \theta\right)\right)\left(m\left(W_{i}, \theta\right)-E m\left(W_{i}, \theta\right)\right)$ to be i.i.d. across elements and consider three distributions for the elements: standard normal (i.e., $\mathrm{N}(0,1)$ ), $t_{3}$, and chi-squared with three degrees of freedom $\chi_{3}^{2}$. All of these distributions are centered and scaled to have mean zero and variance one. The power results are "size-corrected" based on the true $\Omega$ matrix. For $p=2$, 4, and 10, we use 5000, 3000, and 1000 critical value and rejection probability repetitions, respectively, for the results under the null and under the alternative. ${ }^{24}$

We note that the finite-sample testing problem for any moment inequality model fits into the framework above for some correlation matrix $\Omega$ and some distribution of $Z^{\dagger}$. Hence, the finite-sample results given here provide a level of generality that usually is lacking with finite-sample simulation results.

The upper part of Table III provides the finite-sample maximum null rejection probabilities (MNRP's) of the nominal .05 normal and bootstrap versions of the AQLR/tTest/ $\kappa$ Auto test as well the bootstrap version of the ELR/t-Test/ $\kappa$ Auto test. The MNRP is the maximum rejection probability over mean vectors $\mu$ in the null hypothesis for a given correlation matrix $\Omega$ and a given distribution of $Z^{\dagger}$. The lower part of Table

\footnotetext{
${ }^{24}$ The binding constraint on the number of simulation repetitions is the ELR test, see below for details.
} 
III provides MNRP-corrected finite-sample average power for the same three tests. The average power results are for the same mean vectors $\mu$ in the alternative hypothesis as considered above for asymptotic power.

Table III shows that the AQLR/t-Test/ $\kappa$ Auto bootstrap test performs well with MNRP's in the range of $[.043, .066]$. In contrast, the AQLR normal test over-rejects somewhat for some $\Omega$ matrices with the normal and $t_{3}$ distributions for which its MNRP's are in the range of $[.045, .092]$. With the skewed distribution, $\chi_{3}^{2}$, the AQLR normal test over-rejects the null hypothesis substantially with its MNRP's being in the range $[.068, .153]$. The fact that over-rejection is largest for a skewed distribution is not surprising because the first term in the Edgeworth expansion of a sample average is a skewness term and the statistics considered here are simple functions of sample averages.

The ELR bootstrap test performs similarly to the AQLR bootstrap test in terms of null rejection probabilities. Its average amount of over-rejection over the 27 cases is .012, whereas it is .005 for the AQLR bootstrap test.

For the $\mathrm{N}(0,1), t$, and $\chi_{3}^{2}$ distributions, Table III shows that the AQLR bootstrap test has finite-sample average power compared to the AQLR normal test that is similar, inferior, and superior, respectively.

The ELR bootstrap test performs similarly to the AQLR bootstrap test in terms of power. Computation of the ELR/t-Test/ $\kappa$ Auto bootstrap test using $R=10,000$ simulation repetitions takes $9.5,11.8,16.2,31.9,59.0$, and 182.7 seconds when $p=2,4,10,20$, 30 , and 50, respectively, and $n=250$ using GAUSS on a PC with a $3.4 \mathrm{GHz}$ processor. This is slower than the AQLR/t-Test/ $\kappa$ Auto bootstrap test (see Section 2) by a factor of 4.4 to 7.7 .

AJ2 reports additional finite-sample results for the case of singular correlation matrices. The results for the AQLR/t-Test/ $/$ Auto test show that the bootstrap version performs better than the normal version in terms of MNRP's but similarly in terms of average power. Both tests perform well in an absolute sense. The bootstrap version of the MP-QLR/t-Test/ $\kappa$ Auto test also is found to have good MNRP's. However, its finite-sample average power is much inferior to that of the AQLR/t-Test/ $\kappa$ Auto bootstrap test-quite similar to the asymptotic power differences.

For the ELR/t-Test/ $\kappa$ Auto bootstrap test, results for singular correlation matrices are reported in AJ2 only for the case of $p=2$. The reason is that with a singular correlation matrix, the Hessian of the empirical likelihood objective function is singular a.s., which causes difficulties for standard derivative-based optimization algorithms 
when computing the ELR test statistic. With $p=4$ and $p=10$, the constrained optimization algorithm in GAUSS exhibits convergence problems and computation times are prohibitively large. For $p=2$, the ELR bootstrap test's performance is essentially the same as that of the AQLR bootstrap test in terms of MNRP's and power.

In conclusion, we find that the AQLR/t-test/ $\kappa$ Auto bootstrap test, which is the recommended test, performs well in an absolute sense with both nonsingular and singular variance matrices and out-performs the other tests considered in terms of asymptotic and finite-sample MNRP's or power, computational time, and/or computational stability.

\section{References}

Andrews, D. W. K. (1999): "Consistent Moment Selection Procedures for Generalized Method of Moments Estimation," Econometrica, 67, 543-564.

Andrews, D. W. K. and P. Guggenberger (2009): "Validity of Subsampling and "Plug-in Asymptotic" Inference for Parameters Defined by Moment Inequalities," Econometric Theory, 25, 669-709.

Andrews, D. W. K. and P. Jia (2008): "Supplemental Material to 'Inference for Parameters Defined by Moment Inequalities: A Recommended Moment Selection Procedure'," unpublished manuscript, Cowles Foundation, Yale University.

Andrews, D. W. K. and G. Soares (2010): "Inference for Parameters Defined by Moment Inequalities Using Generalized Moment Selection," Econometrica, 78, 119157.

Bugni, F. A. (2010): "Bootstrap Inference in Partially Identified Models Defined by Moment Inequalities: Coverage of the Identified Set," Econometrica, 78, 735-753.

Canay, I. A. (2010): "EL Inference for Partially Identified Models: Large Deviations Optimality and Bootstrap Validity," Journal of Econometrics, 156, 408-425.

Chernozhukov, V., H. Hong, and E. Tamer (2007): "Estimation and Confidence Regions for Parameter Sets in Econometric Models," Econometrica, 75, 1243-1284.

Fan, Y. and S. Park (2007): "Confidence Sets for Some Partially Identified Parameters," unpublished manuscript, Department of Economics, Vanderbilt University. 
Imbens, G. and C. F. Manski (2004): "Confidence Intervals for Partially Identified Parameters," Econometrica, 72, 1845-1857.

Pakes, A., J. Porter, K. Ho, and J. Ishii (2004): "Applications of Moment Inequalities," unpublished working paper, Department of Economics, Harvard University.

Romano, J. P. and A. M. Shaikh (2008): "Inference for Identifiable Parameters in Partially Identified Econometric Models," Journal of Statistical Inference and Planning (Special Issue in Honor of T. W. Anderson), 2786-2807.

Rosen, A. M. (2008): "Confidence Sets for Partially Identified Parameters That Satisfy a Finite Number of Moment Inequalities," Journal of Econometrics, 146, 107-117. 
Table I. Moment Selection Tuning Parameters $\kappa(\delta)$ and Size-Correction Factors $\eta_{1}(\delta)$ and $\eta_{2}(p)$ for $\alpha=.05^{1}$

\begin{tabular}{|c|c|c|c|c|c|c|c|c|c|c|}
\hline$\delta$ & $\kappa(\delta)$ & $\eta_{1}(\delta)$ & & $\delta$ & $\kappa(\delta)$ & $\eta_{1}(\delta)$ & & $\delta$ & $\kappa(\delta)$ & $\eta_{1}(\delta)$ \\
\hline$[-1,-.975)$ & 2.9 & .025 & & {$[-.30,-.25)$} & 2.1 & .111 & & {$[.45, .50)$} & 0.8 & .023 \\
\hline$[-.975,-.95)$ & 2.9 & .026 & & {$[-.25,-.20)$} & 2.1 & .082 & & {$[.50, .55)$} & 0.6 & .033 \\
\hline$[-.95,-.90)$ & 2.9 & .021 & & {$[-.20,-.15)$} & 2.0 & .083 & & {$[.55, .60)$} & 0.6 & .013 \\
\hline$[-.90,-.85)$ & 2.8 & .027 & & {$[-.15,-.10)$} & 2.0 & .074 & & {$[.60, .65)$} & 0.4 & .016 \\
\hline$[-.85,-.80)$ & 2.7 & .062 & & {$[-.10,-.05)$} & 1.9 & .082 & & {$[.65, .70)$} & 0.4 & .000 \\
\hline$[-.80,-.75)$ & 2.6 & .104 & & {$[-.05, .00)$} & 1.8 & .075 & & {$[.70, .75)$} & 0.2 & .003 \\
\hline$[-.75,-.70)$ & 2.6 & .103 & & {$[.00, .05)$} & 1.5 & .114 & & {$[.75, .80)$} & 0.0 & .002 \\
\hline$[-.70,-.65)$ & 2.5 & .131 & & {$[.05, .10)$} & 1.4 & .112 & & {$[.80, .85)$} & 0.0 & .000 \\
\hline$[-.65,-.60)$ & 2.5 & .122 & & {$[.10, .15)$} & 1.4 & .083 & & {$[.85, .90)$} & 0.0 & .000 \\
\hline$[-.60,-.55)$ & 2.5 & .113 & & {$[.15, .20)$} & 1.3 & .089 & & {$[.90, .95)$} & 0.0 & .000 \\
\hline$[-.55,-.50)$ & 2.5 & .104 & & {$[.20, .25)$} & 1.3 & .058 & & {$[.95, .975)$} & 0.0 & .000 \\
\hline$[-.50,-.45)$ & 2.4 & .124 & & {$[.25, .30)$} & 1.2 & .055 & & {$[.975, .99)$} & 0.0 & .000 \\
\hline$[-.45,-.40)$ & 2.2 & .158 & & {$[.30, .35)$} & 1.1 & .044 & & {$[.99,1.0]$} & 0.0 & .000 \\
\hline$[-.40,-.35)$ & 2.2 & .133 & & {$[.35, .40)$} & 1.0 & .040 & & & & \\
\hline$[-.35,-.30)$ & 2.1 & .138 & & {$[.40, .45)$} & 0.8 & .051 & & & & \\
\hline$p$ & & 2 & 3 & 4 & 5 & 6 & 7 & 8 & 9 & 10 \\
\hline$\eta_{2}(p)$ & & .00 & .15 & .17 & .24 & .31 & .33 & .37 & .45 & .50 \\
\hline
\end{tabular}

${ }^{1}$ The values in Table I are obtained by simulating asymptotic formulae using 40,000 critical-value and 40,000 rejection-probability simulation repetitions, see AJ2 for details. 
Table II. Asymptotic Average Power Comparisons (Size-Corrected): MMM, Max, SumMax, \& AQLR Statistics, \& PA, t-Test, $\varphi^{(2)}, \varphi^{(3)}, \varphi^{(4)}, \&$ MMSC Critical Values with $\kappa=$ Best $^{1}$

\begin{tabular}{|c|c|c|c|c|c|c|c|c|c|c|c|}
\hline \multirow[b]{2}{*}{ Stat. } & \multirow{2}{*}{$\begin{array}{l}\text { Crit. } \\
\text { Val. }\end{array}$} & \multirow{2}{*}{$\begin{array}{l}\text { Tuning } \\
\text { Par. } \kappa\end{array}$} & \multicolumn{3}{|c|}{$p=10$} & \multicolumn{3}{|c|}{$p=4$} & \multicolumn{3}{|c|}{$p=2$} \\
\hline & & & $\Omega_{N e g}$ & $\Omega_{\text {Zero }}$ & $\Omega_{\text {Pos }}$ & $\Omega_{N e g}$ & $\Omega_{\text {Zero }}$ & $\Omega_{\text {Pos }}$ & $\Omega_{N e g}$ & $\Omega_{\text {Zero }}$ & $\Omega_{\text {Pos }}$ \\
\hline MMM & PA & - & .04 & .36 & .34 & .20 & .53 & .45 & .48 & .62 & .59 \\
\hline MMM & $t$-Test & Best & .18 & .67 & .79 & .31 & .69 & .76 & .51 & .69 & .72 \\
\hline Max & PA & - & .19 & .44 & .70 & .30 & .57 & .71 & .48 & .64 & .66 \\
\hline Max & $t$-Test & Best & .25 & .58 & .82 & .35 & .66 & .78 & .51 & .69 & .72 \\
\hline SumMax & PA & - & .10 & .43 & .62 & .20 & .55 & .60 & .48 & .62 & .59 \\
\hline SumMax & $t$-Test & Best & .20 & .65 & .81 & .31 & .69 & .77 & .51 & .69 & .72 \\
\hline AQLR & PA & - & .35 & .36 & .69 & .46 & .53 & .70 & .58 & .69 & .65 \\
\hline AQLR & $t$-Test & Best & .55 & .67 & .82 & .60 & .69 & .78 & .65 & .69 & .73 \\
\hline AQLR & $t$-Test & Auto & .55 & .67 & .82 & .59 & .69 & .78 & .65 & .69 & .73 \\
\hline AQLR & $\varphi^{(2)}$ & Best & $.51^{\dagger}$ & $.65^{\dagger}$ & $.81^{\dagger}$ & $.60^{\diamond}$ & $.69^{*}$ & $.78^{*}$ & $.66^{*}$ & $.69^{*}$ & $.72^{*}$ \\
\hline AQLR & $\varphi^{(3)}$ & Best & $.43^{\dagger}$ & $.63^{\dagger}$ & $.81^{\dagger}$ & $.55^{\diamond}$ & $.68^{*}$ & $.78^{*}$ & $.61^{*}$ & $.69^{*}$ & $.72^{*}$ \\
\hline AQLR & $\varphi^{(4)}$ & Best & $.51^{\dagger}$ & $.65^{\dagger}$ & $.81^{\dagger}$ & $.60^{\diamond}$ & $.70^{*}$ & $.78^{*}$ & $.66^{*}$ & $.69^{*}$ & $.72^{*}$ \\
\hline AQLR & MMSC & Best & $.56^{\dagger}$ & $.66^{\dagger}$ & $.81^{\dagger}$ & .63 & .69 & .78 & .65 & .69 & .73 \\
\hline Power & Envelope & - & .85 & .85 & .85 & .80 & .80 & .80 & .75 & .75 & .75 \\
\hline
\end{tabular}

${ }^{1} \kappa=$ Best denotes the $\kappa$ value that maximizes asymptotic average power. All cases not marked with $\mathrm{a}^{*}, \diamond$, or ${ }^{\dagger}$ are based on $(40,000,40,000,40,000)$ critical-value, sizecorrection, and power repetitions, respectively.

${ }^{*}$ Results are based on $(5000,5000,5000)$ repetitions.

$\diamond$ Results are based on $(2000,2000,2000)$ repetitions.

${ }^{\dagger}$ Results are based on $(1000,1000,1000)$ repetitions. 
Table III. Finite-Sample Maximum Null Rejection Probabilities (MNRP's) and ("SizeCorrected") Average Power of the Nominal .05 AQLR/t-Test/ $\kappa$ Auto Test with Normal $(\mathrm{AQLR} / \mathrm{Nm})$ and Bootstrap-Based (AQLR/Bt) Critical Values and ELR/t-Test/ $\kappa$ Auto Test with Bootstrap-Based (ELR/Bt) Critical Values

\begin{tabular}{|c|c|c|c|c|c|c|c|c|c|c|c|}
\hline \multirow[b]{2}{*}{ Test } & \multirow[b]{2}{*}{ Dist } & \multirow[b]{2}{*}{$\mathrm{H}_{0} / \mathrm{H}_{1}$} & \multicolumn{3}{|c|}{$p=10$} & \multicolumn{3}{|c|}{$p=4$} & \multicolumn{3}{|c|}{$p=2$} \\
\hline & & & $\Omega_{N e g}$ & $\Omega_{\text {Zero }}$ & $\Omega_{\text {Pos }}$ & $\Omega_{N e g}$ & $\Omega_{\text {Zero }}$ & $\Omega_{\text {Pos }}$ & $\Omega_{N e g}$ & $\Omega_{\text {Zero }}$ & $\Omega_{P o s}$ \\
\hline AQLR/Nm & $\mathrm{N}(0,1)$ & $\mathrm{H}_{0}$ & .088 & .092 & .057 & .065 & .062 & .049 & .056 & .058 & .053 \\
\hline $\mathrm{AQLR} / \mathrm{Bt}$ & $\mathrm{N}(0,1)$ & $\mathrm{H}_{0}$ & .061 & .062 & .058 & .053 & .056 & .049 & .054 & .053 & .052 \\
\hline $\mathrm{ELR} / \mathrm{Bt}$ & $\mathrm{N}(0,1)$ & $\mathrm{H}_{0}$ & .075 & .076 & .073 & .059 & .065 & .054 & .055 & .058 & .053 \\
\hline AQLR/Nm & $t_{3}$ & $\mathrm{H}_{0}$ & .059 & .067 & .045 & .050 & .049 & .047 & .053 & .047 & .046 \\
\hline AQLR/Bt & $t_{3}$ & $\mathrm{H}_{0}$ & .043 & .055 & .055 & .051 & .058 & .052 & .057 & .055 & .056 \\
\hline $\mathrm{ELR} / \mathrm{Bt}$ & $t_{3}$ & $\mathrm{H}_{0}$ & .059 & .072 & .072 & .056 & .067 & .058 & .057 & .057 & .055 \\
\hline AQLR/Nm & $\chi_{3}^{2}$ & $\mathrm{H}_{0}$ & .136 & .153 & .068 & .093 & .101 & .062 & .085 & .087 & .080 \\
\hline AQLR/Bt & $\chi_{3}^{2}$ & $\mathrm{H}_{0}$ & .062 & .066 & .057 & .050 & .055 & .050 & .054 & .053 & .056 \\
\hline $\mathrm{ELR} / \mathrm{Bt}$ & $\chi_{3}^{2}$ & $\mathrm{H}_{0}$ & .068 & .077 & .065 & .054 & .061 & .054 & .053 & .054 & .055 \\
\hline AQLR/Nm & $\mathrm{N}(0,1)$ & $\mathrm{H}_{1}$ & .45 & .59 & .78 & .54 & .63 & .76 & .63 & .68 & .71 \\
\hline AQLR/Bt & $\mathrm{N}(0,1)$ & $\mathrm{H}_{1}$ & .46 & .62 & .77 & .54 & .64 & .76 & .63 & .68 & .71 \\
\hline $\mathrm{ELR} / \mathrm{Bt}$ & $\mathrm{N}(0,1)$ & $\mathrm{H}_{1}$ & .49 & .61 & .76 & .56 & .64 & .75 & .63 & .68 & .71 \\
\hline AQLR/Nm & $t_{3}$ & $\mathrm{H}_{1}$ & .58 & .69 & .84 & .66 & .76 & .81 & .70 & .76 & .72 \\
\hline AQLR/Bt & $t_{3}$ & $\mathrm{H}_{1}$ & .56 & .67 & .79 & .61 & .71 & .78 & .67 & .72 & .71 \\
\hline $\mathrm{ELR} / \mathrm{Bt}$ & $t_{3}$ & $\mathrm{H}_{1}$ & .55 & .62 & .76 & .61 & .67 & .76 & .64 & .68 & .71 \\
\hline AQLR/Nm & $\chi_{3}^{2}$ & $\mathrm{H}_{1}$ & .37 & .42 & .72 & .48 & .53 & .71 & .56 & .57 & .61 \\
\hline $\mathrm{AQLR} / \mathrm{Bt}$ & $\chi_{3}^{2}$ & $\mathrm{H}_{1}$ & .43 & .51 & .72 & .53 & .57 & .70 & .57 & .59 & .62 \\
\hline $\mathrm{ELR} / \mathrm{Bt}$ & $\chi_{3}^{2}$ & $\mathrm{H}_{1}$ & .41 & .47 & .70 & .53 & .56 & .70 & .56 & .59 & .62 \\
\hline
\end{tabular}

